\section{Pediatric weight estimation: validation of the PAWPER XL tape and the PAWPER XL tape mid-arm circumference method in a South African hospital}

\author{
Ming-Tung Wu, Mike Wells \\ Division of Emergency Medicine, Faculty of Health Sciences, University of the Witwatersrand, \\ Johannesburg, South Africa
}

Objective The primary aim of this study was to prospectively compare the performance of the Broselow tape, Mercy method, pediatric advanced weight prediction in the emergency room extra-long (PAWPER XL) tape, and PAWPER XL mid-arm circumference (MAC) method in estimating the weight of children from a low-income setting. The secondary aim was to analyze the time taken to perform each method.

Methods This analyzed a convenience study sample of 300 children aged 0 to 18 years at the Baragwanath Hospital in South Africa. Weight estimations were obtained using each of the weight estimation systems on each child. These weight estimations were then compared against the actual weight to determine bias, precision, and accuracy of the estimation methods.

Results The PAWPER XL tape and PAWPER XL-MAC methods performed the best and provided estimated weights within $10 \%$ of the actual weight in $62.7 \%$ and $67.3 \%$ of cases, respectively, followed by the Mercy method (56.5\%) and Broselow tape (43.9\%). The use of MAC improved the accuracy of estimation, especially in heavier and taller children. The median times taken to perform measurements using the Broselow tape, Mercy method, PAWPER XL tape, and PAWPER XL-MAC method were 11.3 seconds, 34.7 seconds, 9.3 seconds, and 33.9 seconds respectively.

Conclusion The PAWPER XL tape and PAWPER XL-MAC methods were the most accurate methods of estimating weight in this group of children. These methods may be considered in preference to the Broselow tape or the Mercy method for emergency weight estimation in low socioeconomic status populations.

Keywords Resuscitation; Emergency treatment; Devices, medical
eISSN: 2383-4625

Received: 23 September 2019

Revised: 12 November 2019

Accepted: 13 November 2019

Correspondence to: Mike Wells Division of Emergency Medicine, Faculty of Health Sciences, University of the Witwatersrand, 7 York Road, Parktown, Johannesburg 2193, South Africa

E-mail:mike.wells@ emergencymedicine.co.za ORCID https://orcid.org/0000-0002-4520-2007

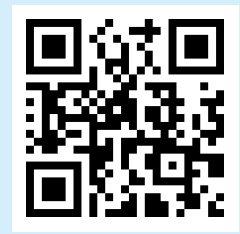

How to cite this article:

Wu MT, Wells M. Pediatric weight estimation: validation of the PAWPER XL tape and the PAWPER XL tape mid-arm circumference method in a South African hospital. Clin Exp Emerg Med 2020;7(4):290-301. https://doi. org/10.15441/ceem.19.082

This is an Open Access article distributed under the terms of the Creative Commons Attribution Non-Commercial License (https:// creativecommons.org/licenses/by-nc/4.0/). 


Capsule
What is already known
The use of dual length-and habitus-based methods of weight estimation have been shown to be the most accurate of
all systems. One such system, PAWPER XL mid-arm circumference (MAC) method showed a very high accuracy in "vir-
tual" database studies but needed to be validated in a prospective study.
What is new in the current study
The PAWPER XL-MAC method was the most accurate and outperformed the Broselow tape and the Mercy method in
this study. All weight estimation systems were able to be used within a clinically appropriate time, although the meth-
ods requiring a measurement of MAC, such as the PAWPER XL-MAC and Mercy methods, took slightly longer than the
other methods.

\section{INTRODUCTION}

The management directed at children is generally quantified by their body weight. The actual body weight of children presenting in emergency settings or receiving critical care is often difficult or impossible to obtain. ${ }^{1}$ This is either due to the need for immediate intervention and resuscitation or due to immobilization. As the estimated body weight determines the efficacy and safety of therapeutic interventions, this estimation needs to be accurate. ${ }^{1,2}$

Various length-based weight prediction methods have been developed for children in the past, the most well-known of which is the Broselow tape. This tape is commonly used in the emergency setting because of its simplicity and immediate availability of medication dosages and equipment sizes. ${ }^{3}$ However, it also has significant disadvantages. Many studies have shown that the Broselow tape overestimates children's weights in lower socioeconomic settings and underestimates the weight of children from higher income countries. ${ }^{4-7}$ This can potentially lead to a dangerous degree of drug overdosing or underdosing.

Other than parental estimates, the most accurate current methods of weight estimation are the newer two-dimensional dual length- and body habitus-based systems, such as the Mercy method and the pediatric advanced weight prediction in the emergency room (PAWPER) tape system. ${ }^{8-10}$ The Mercy method uses surrogates of the total body length (humeral length) and body habitus (mid-arm circumference, MAC) to estimate weight. This method has been shown to have good accuracy in populations with a high prevalence of obesity or of underweight children.11-14 The PAWPER tape system makes use of a child's body length and a general impression (gestalt visual assessment system) or a figural reference image rating scale (using pictures of children at each habitus score [HS]) to assess habitus and provide a weight estimation from the tape. ${ }^{15}$ This system is potentially vulnerable to error as different health care workers may have different percep- tions of body habitus. ${ }^{16}$ For this reason, the PAWPER extra-long $(\mathrm{XL})-\mathrm{MAC}$ system was developed, which makes use of MAC to define body habitus, rather than a gestalt impression. ${ }^{17}$ Since MAC has previously been shown to be strongly associated with body habitus and is an objective measurement, as opposed to an observer's impression of a patient, its use may increase the accuracy of the PAWPER XL-MAC system when compared to the original system. ${ }^{18,19}$ However, measuring MAC might take longer to produce a weight estimate, which would not be ideal during emergency care. Preliminary validation studies conducted from datasets of anthropometric data have shown the excellent accuracy of the PAWPER XL-MAC system, on par with the regular PAWPER XL tape, but no prospective studies have yet evaluated this method. ${ }^{19,20}$

With the above background, the primary aim of this study was to evaluate the weight estimation accuracy and time to obtain weight estimation for the PAWPER XL tape and the PAWPER XLMAC method of children from a low socioeconomic background. The secondary aim was to compare the performance of these methods with that of the Mercy method and the Broselow tape (as a past reference standard).

\section{METHODS}

\section{Ethics statement}

This study was approved by the Human Research Ethics Committee of the Faculty of Health Science of the University of the Witwatersrand (M161141). Informed consent was obtained from the parent or legal guardian, and assent was obtained from children who were above 7 years of age.

\section{Study design}

This was a prospective cross-sectional study. The study was conducted from June 2017 to September 2017. 


\section{Study site}

The study took place at Baragwanath Hospital in Soweto, one of the biggest townships in South Africa, situated to the south-west of Johannesburg. Many parts of Soweto rank among the poorest in South Africa.

\section{Study population}

The study population comprised children from birth to 18 years old who presented to the pediatric unit (outpatients and inpatients), otolaryngology clinic, and ophthalmology clinic.

All children who were not in need of emergency medical treatment were eligible for inclusion into the study. The specific exclusion criteria were as follows: children whose length could not be

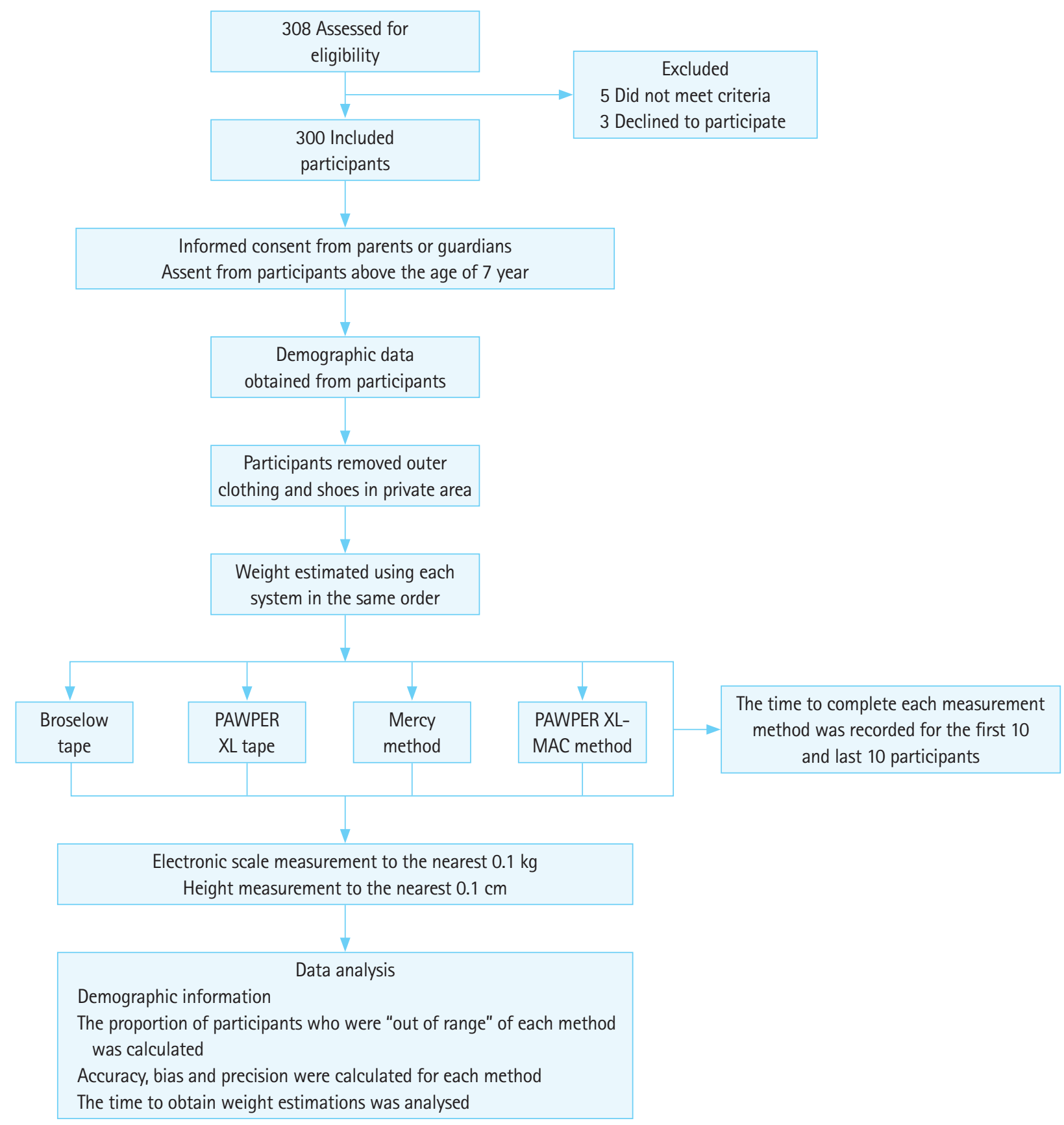

Fig. 1. Flow chart for the study data collection procedure and data analysis. The study included 300 children, and all measurements were performed on each of these participants. Additional measurements of the time taken to complete the weight estimations were performed only in the first 10 and last 10 participants. PAWPER XL, pediatric advanced weight prediction in the emergency room extra-long; MAC, mid-arm circumference. 
accurately assessed (e.g., presence of contractures); failure to obtain assent from the child or consent from either guardian or parents; children who could not be weighed on a scale; and children with any known underlying conditions that would produce abnormal body composition (e.g., edema) (Fig. 1).

A non-consecutive convenience sample of 300 children was used. This sample size was sufficient to detect a 10\% relative difference in accuracy between the weight estimation systems, at a power of $80 \%$, within an accuracy range of $50 \%$ to $80 \%$.

\section{Weight estimation methods evaluated in this study}

The Broselow tape is a length-based tape (which includes precalculated drug doses) and is one of the most studied weight estimation methods worldwide.? It is used by laying the tape alongside the child and aligning the start of the tape with the top of the child's head. The weight estimation can then be read directly off the tape at the length where the heel crosses the tape. The Broselow tape can only provide weight estimations in children less than $143 \mathrm{~cm}$ in length.

The Mercy method is a dual length- and habitus-based method of weight estimation. ${ }^{21}$ Measurements are taken of the child's humeral length and MAC using a regular anthropometric tape. These measurements are used to identify 'partial weights' from a table (one for the humeral length and one for the MAC) which are added together to obtain the weight estimation. Recently, this method is one of the most accurate pediatric weight estimation methods in the world. ${ }^{2}$

The PAWPER XL tape system is also a dual length- and habitusbased method of weight estimation (Fig. 2). ${ }^{15}$ The tape is placed alongside the child, with the start of the tape aligned to the top of the child's head. The length segment on the tape where the child's heel crosses the tape is then used for the weight estimation. The child's weight estimate can be adjusted up or down from "normal weight" based on their body habitus using the HS system. Using a card with reference images for guidance, the user assigns an HS from 1 to 7 for the child. An HS of 1 would represent an underweight child, an HS of 3 a normal-weight child, an HS of 5 an obese child, and an HS of 7 a severely obese child. Each HS corresponds to a predicted weight within each length segment of the tape, which can be read off by the user. The PAWPER XL tape can provide weight estimations in children up to $180 \mathrm{~cm}$ in length. At present, it is also one of the most accurate weight estimation systems available globally. ${ }^{2,10}$

The PAWPER XL-MAC method is used in the same way as the regular PAWPER XL tape, except that the HS is determined from measurements of MAC (Fig. 2). ${ }^{16,19}$ The tape presents MAC measurement ranges for each $\mathrm{HS}$ within each length segment of the

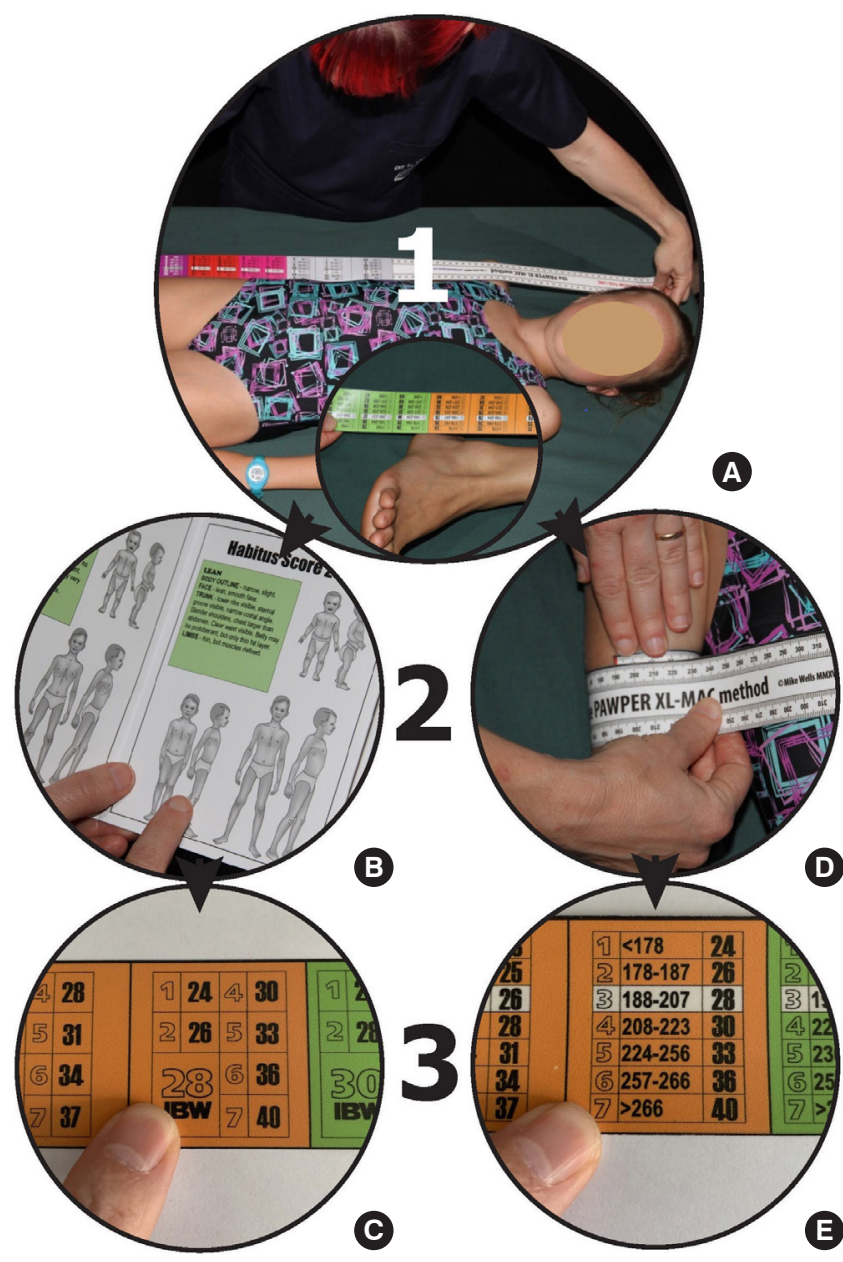

Fig. 2. Steps in using the PAWPER XL tape and the PAWPER XL midarm circumference (MAC) method. For the PAWPER XL tape, (A) step 1: the tape is used to measure the child's length from the top of the head to where the tape crosses the heel (this is the same for both systems). The user must take note of the weight segment into which the child falls. (B) Step 2: the user assesses the child's habitus using a gestalt impression or with the aid of a habitus-assessment card. (C) Step 3: the predicted weight is read off from the tape itself (bold numbers) corresponding to the assigned habitus score. For the PAWPER XL-MAC system, (A) step 1: the tape is used to measure the child's length from the top of the head to where the tape crosses the heel. The user must take note of the weight segment into which the child belongs. (D) Step 2: the user measures the child's MAC using the tape. (E) Step 3: the predicted weight is read off the tape (bold numbers) from the habitus score category that was determined by the MAC measurements. The cut-off values are shown on the tape. Written informed consent was obtained for the use of the model portrayed in these images.

PAWPER XL, pediatric advanced weight prediction in the emergency room extra-long.

tape. This allows the user to determine the appropriate HS from the child's MAC. The corresponding predicted weights can then be read directly off the tape. 


\section{Study procedure}

Fig. 1 shows the study protocol and procedures in obtaining the weight estimations. Fig. 2 provides a description of the use of the PAWPER XL tape and the PAWPER XL-MAC method. A digital electronic scale was used to obtain the participants' actual weight (DQUIP Smart Scale, EF- 8 Series; DQUIP, Johannesburg, South Africa). Participants who were unable to stand were carried by the data collector. Then, while carrying the child, data collector stood on the electronic scale. The weight of the data collector was subtracted from the total weight to obtain the participant's actual weight. The time to complete each measurement method was recorded for the first 10 and last 10 participants. This was done to determine the effect of training and practice on the weight estimation times.

All measurements were made by a single data collector (MTW). To minimize bias, the procedures were carried out in the same order for each child, and the data collector was blinded to the actual weight until all other estimations had been obtained. Time measurements were also performed in the exactly the same way for each weight estimation method for the subgroup of children.

\section{Data analysis}

The performances of the weight estimation methods were compared with the actual weight and against each other. Analyses of the entire study population, as well as for subgroups of weight $(\leq 10,10.1-20,20.1-40,>40 \mathrm{~kg})$, age $(<2,2-5,5.1-10,>10$ years), and body HS (HS $<3, \mathrm{HS} 3$ and HS4, HS $>4$ ) were performed.

The primary outcome measures were as follows: mean percentage error was calculated from the difference between the predicted weight and the actual weight. This gave a measurement of estimation bias; root mean squared percentage error was also calculated as a measure of precision. It is the average of the absolute (root-squared) percentage errors for each weight estimation; the number of cases that fell within 10\% (PW10) and 20\% (PW20) of the actual weight. This provided a measure of overall accuracy of the methods and was regarded as the primary indicator of overall performance.

\section{Outcome measures}

The primary endpoint was the performance of the PAWPER XL method and the PAWPER XL-MAC method in pediatric weight estimation and comparison with other weight prediction methods in a study sample from a public hospital in South Africa. The secondary endpoint was the time taken to perform each method and any improvement in time with increasing practice.

\section{Statistical analysis}

All data were captured from the data collection sheets and entered onto an electronic spreadsheet (Microsoft Excel for MAC 2011; Microsoft Corporation, Redmond, WA, USA). Statistical analysis was performed with Stata ver. 14 (StataCorp., College Station, TX, USA) for basic descriptive statistics and statistical comparisons.

Weight estimation data were described and analyzed using the following statistics: mean percentage difference with 95\% limits of agreement-this represented the bias or trueness of the weight estimation system; the root mean squared percentage error-this represented the overall precision; and the PW10 and PW20-this represented overall accuracy.

\section{RESULTS}

A total of 300 children participated in the study. The study included 164 boys (54.7\%) and 136 girls (45.3\%). The median and in-

Table 1. Demographic and anthropometric data for the study population

\begin{tabular}{|c|c|c|c|c|c|c|c|c|}
\hline & \multirow{2}{*}{ All } & \multicolumn{4}{|c|}{ Characteristics of population by subgroup of weight category } & \multicolumn{3}{|c|}{ Characteristics of population by subgroup of habitus } \\
\hline & & $\leq 10 \mathrm{~kg}$ & $10.1-20 \mathrm{~kg}$ & $20.1-40 \mathrm{~kg}$ & $\geq 40 \mathrm{~kg}$ & $\mathrm{HS}<3$ & HS 3-4 & $\mathrm{HS}>4$ \\
\hline Total & 300 & $74(25)$ & $118(39)$ & $75(25)$ & $33(11)$ & $82(27)$ & $158(53)$ & $60(20)$ \\
\hline Female & 136 & 38 (27.9) & $50(36.8)$ & $31(22.8)$ & $17(12.5)$ & $40(29.4)$ & $66(48.5)$ & $30(22.1)$ \\
\hline Male & 164 & $36(22.0)$ & $68(41.5)$ & $44(26.8)$ & $16(9.7)$ & $42(25.6)$ & $92(56.1)$ & $30(18.3)$ \\
\hline Age $(y r)$ & $4.0(1.3-8.0)$ & $0.5(0.1-1.3)$ & $3(2.0-5.0)$ & $8(7-10)$ & $13(11-16)$ & $7(4-10)$ & $2(1.6-8)$ & $2(1.3-6.3)$ \\
\hline Weight (kg) & $16.0(10.2-26.9)$ & $6.7(4.9-8.4)$ & $14.9(12.5-17.1)$ & $27.9(23.5-31.6)$ & $49.8(44.2-59.9)$ & $20.6(14.0-29.7)$ & $14.6(9.3-23.9)$ & $14.9(9.8-20.7)$ \\
\hline $\begin{array}{l}\text { Z-score } \\
\text { (BMI-for-age) }\end{array}$ & $-0.8(-1.9-0.4)$ & $-1.0(-1.9-0.2)$ & $-1.0(-2.3-0.1)$ & $-0.7(-1.4-0.6)$ & $0.3(-0.8-1.8)$ & $-2.4(-3.3--1.2)$ & $-1.3(-2.7-0.0)$ & $0.3(-0.6-1.6)$ \\
\hline
\end{tabular}

Values are presented as number (\%) or median (interquartile range). A HS of $<3$ indicates an underweight child, habitus score of 3 and 4 indicate normal-weight children, and higher habitus scores indicate overweight and obese children.

HS, habitus score; BMI, body mass index. 
Table 2. Performance data for all methods of weight estimation

\begin{tabular}{|c|c|c|c|c|c|}
\hline & & $\begin{array}{l}\text { Broselow } \\
\text { tape }\end{array}$ & $\begin{array}{l}\text { Mercy } \\
\text { method }\end{array}$ & $\begin{array}{l}\text { PAWPER XL } \\
\text { tape }\end{array}$ & $\begin{array}{l}\text { PAWPER } \\
\text { XL-MAC } \\
\text { method }\end{array}$ \\
\hline \multirow{6}{*}{$\begin{array}{l}\text { All } \\
\qquad(n=300)\end{array}$} & MPE & 8.5 & 6.9 & 3.3 & 6.2 \\
\hline & RMSPE (SD) & 14.4 (11.5) & $11.9(13.4)$ & $10.4(8.8)$ & $9.6(8.2)$ \\
\hline & PW10 & 43.9 & 56.5 & 62.7 & 67.3 \\
\hline & PW20 & 73.9 & 84.3 & 88.3 & 88.7 \\
\hline & LLOA & -22.1 & -25.2 & -21.4 & -13.9 \\
\hline & ULOA & 39.6 & 39.5 & 28.5 & 26.7 \\
\hline \multirow{6}{*}{$\begin{array}{l}\leq 10 \mathrm{~kg} \\
\quad(n=74)\end{array}$} & MPE & 10.5 & 20.4 & 4.1 & 9.5 \\
\hline & RMSPE (SD) & $15.4(13.8)$ & $22.5(20.6)$ & $12.3(10.4)$ & $14.0(9.8)$ \\
\hline & PW10 & 41.7 & 28.8 & 52.1 & 42.5 \\
\hline & PW20 & 73.6 & 60.3 & 79.5 & 71.2 \\
\hline & LLOA & -27.9 & -27.6 & -30.1 & -23.6 \\
\hline & ULOA & 47.1 & 65.7 & 36.7 & 40.9 \\
\hline \multirow{6}{*}{$\begin{array}{c}10.1-20 \mathrm{~kg} \\
(\mathrm{n}=118)\end{array}$} & MPE & 9.1 & 4.1 & 3.9 & 7.8 \\
\hline & RMSPE(SD) & $13.3(10.4)$ & $9.5(7.9)$ & $9.2(6.9)$ & $9.4(6.4)$ \\
\hline & PW10 & 46.6 & 60.2 & 66.1 & 68.6 \\
\hline & PW20 & 76.3 & 89.0 & 94.9 & 93.2 \\
\hline & LLOA & -18.9 & -18.7 & -17.4 & -8.6 \\
\hline & ULOA & 37.1 & 26.9 & 25.2 & 24.1 \\
\hline \multirow{6}{*}{$\begin{array}{c}20.1-40 \mathrm{~kg} \\
(\mathrm{n}=75)\end{array}$} & MPE & 8.3 & 1.7 & 4.7 & 4.1 \\
\hline & RMSPE (SD) & $13.5(10.1)$ & $7.1(5.6)$ & $9.9(7.8)$ & $6.7(5.0)$ \\
\hline & PW10 & 46.7 & 72.0 & 66.7 & 81.3 \\
\hline & PW20 & 75.0 & 97.3 & 86.7 & 97.3 \\
\hline & LLOA & -22.6 & -17.8 & -20.3 & -12.3 \\
\hline & ULOA & 38.1 & 20.3 & 28.7 & 19.6 \\
\hline \multirow{6}{*}{$\begin{array}{l}\geq 40 \mathrm{~kg} \\
\quad(n=33)\end{array}$} & MPE & 24.3 & 2.5 & -1.8 & 0.5 \\
\hline & RMSPE (SD) & $24.3(5.6)$ & $7.6(7.2)$ & $8.3(6.6)$ & $4.7(3.0)$ \\
\hline & PW10 & 0.0 & 72.7 & 72.7 & 93.9 \\
\hline & PW20 & 33.3 & 93.9 & 97 & 100 \\
\hline & LLOA & -37.7 & -17.8 & -22.5 & -11.7 \\
\hline & ULOA & -10.9 & 22.7 & 19.0 & 12.6 \\
\hline \multirow{6}{*}{$\begin{aligned} & H S<3 \\
&(n=82)\end{aligned}$} & MPE & 22.9 & 4.8 & 11.2 & 6.7 \\
\hline & RMSPE (SD) & $23.6(12.8)$ & $9.3(8.4)$ & $14.5(12.4)$ & $10.7(11.7)$ \\
\hline & PW10 & 10.0 & 63.0 & 39.0 & 62.2 \\
\hline & PW20 & 41.7 & 88.9 & 76.8 & 89.0 \\
\hline & LLOA & -4.8 & -18.0 & -19.4 & -21.6 \\
\hline & ULOA & 50.7 & 27.6 & 41.7 & 35.0 \\
\hline \multirow{6}{*}{$\begin{array}{l}\text { HS 3-4 } \\
\quad(n=158)\end{array}$} & MPE & 8.5 & 7.1 & 2.6 & 5.9 \\
\hline & RMSPE (SD) & $11.3(8.7)$ & $12.4(15.4)$ & $8.2(7.3)$ & $8.9(7.6)$ \\
\hline & PW10 & 55.0 & 57.6 & 77.2 & 72.8 \\
\hline & PW20 & 85.7 & 85.4 & 93.7 & 91.1 \\
\hline & LLOA & -18.0 & -30.7 & -21.8 & -17.9 \\
\hline & ULOA & 33.7 & 43.3 & 25.9 & 28.5 \\
\hline \multirow{6}{*}{$\begin{array}{l}\text { HS }>4 \\
\quad(n=60)\end{array}$} & MPE & -6.0 & 11.1 & -6.2 & 5.9 \\
\hline & RMSPE (SD) & $10.7(8.2)$ & $13.8(12.6)$ & $9.9(7.4)$ & $9.8(7.7)$ \\
\hline & PW10 & 56.6 & 46.7 & 60.0 & 63.3 \\
\hline & PW20 & 83.0 & 76.7 & 93.3 & 85.0 \\
\hline & LLOA & -29.8 & -18.7 & -27.3 & -16.0 \\
\hline & ULOA & 17.8 & 40.8 & 14.8 & 27.7 \\
\hline
\end{tabular}

(continued to the next)
Table 2. Continued

\begin{tabular}{|c|c|c|c|c|c|}
\hline & & $\begin{array}{l}\text { Broselow } \\
\text { tape }\end{array}$ & $\begin{array}{l}\text { Mercy } \\
\text { method }\end{array}$ & $\begin{array}{l}\text { PAWPER XL } \\
\text { tape }\end{array}$ & $\begin{array}{c}\text { PAWPER } \\
\text { XL-MAC } \\
\text { method }\end{array}$ \\
\hline \multirow{6}{*}{$\begin{array}{c}\text { Age }<2 \text { yr } \\
(n=88)\end{array}$} & MPE & 6.5 & 17.6 & 0.8 & 7.3 \\
\hline & RMSPE (SD) & $13.9(11.9)$ & $20.3(19.6)$ & $12.4(13.0)$ & 14.1 (12.8) \\
\hline & PW10 & 46.5 & 36.4 & 58.0 & 48.9 \\
\hline & PW20 & 76.7 & 63.6 & 83.0 & 75.0 \\
\hline & LLOA & -30.9 & -28.8 & -37.6 & -31.0 \\
\hline & ULOA & 42.6 & 61.8 & 37.8 & 44.2 \\
\hline \multirow{7}{*}{$\begin{array}{l}\text { Age } 2-5 y r \\
(n=92)\end{array}$} & MPE & 9.4 & 4.8 & 4.1 & 7.9 \\
\hline & RMSPE (SD) & $12.8(9.6)$ & $9.0(7.7)$ & $8.8(6.1)$ & $8.8(5.6)$ \\
\hline & PW10 & 50.0 & 62.0 & 66.3 & 70.7 \\
\hline & PW20 & 77.2 & 91.3 & 96.7 & 95.7 \\
\hline & LLOA & -17.8 & -18.2 & -17.0 & -7.8 \\
\hline & ULOA & 35.8 & 27.0 & 24.5 & 22.9 \\
\hline & MPE & 11.1 & 1.7 & 6.0 & 5.4 \\
\hline \multirow{5}{*}{$\begin{array}{c}\text { Age } 6-10 \mathrm{yr} \\
(\mathrm{n}=76)\end{array}$} & RMSPE (SD) & $15.9(12.0)$ & $8.1(6.5)$ & $10.4(8.6)$ & $7.7(6.5)$ \\
\hline & PW10 & 36.2 & 65.3 & 64.0 & 76.0 \\
\hline & PW20 & 69.6 & 94.7 & 84.0 & 92.0 \\
\hline & LLOA & -21.2 & -18.6 & -18.0 & -11.4 \\
\hline & ULOA & 43.5 & 21.9 & 30.0 & 22.2 \\
\hline \multirow{6}{*}{$\begin{array}{c}\text { Age }>10 y r \\
(n=44)\end{array}$} & MPE & 14.4 & 2.0 & 1.5 & 1.4 \\
\hline & RMSPE (SD) & 14.4 (9.9) & $7.3(6.8)$ & $9.0(6.3)$ & $5.4(4.4)$ \\
\hline & PW10 & 40.0 & 72.7 & 68.2 & 86.4 \\
\hline & PW20 & 60.0 & 95.5 & 93.2 & 100 \\
\hline & LLOA & -7.3 & -17.3 & -19.9 & -12.1 \\
\hline & ULOA & 36.1 & 21.4 & 23.0 & 15.0 \\
\hline
\end{tabular}

Measures of bias, precision, and accuracy for the study participants are shown for each weight, age, and habitus category. A positive value of the MPE indicates an overestimation of weight.

PAWPER XL, pediatric advanced weight prediction in the emergency room extralong; MAC, mid-arm circumference; MPE, mean percentage error; RMSPE, root mean square percentage error; SD, standard deviation; PW10, percentage of weight estimations falling within 10\% of the actual weight; PW20, percentage of weight estimations falling within $20 \%$ of the actual weight; LLOA, $95 \%$ lower limit of agreement; ULOA, 95\% upper limit of agreement; HS, habitus score.

terquartile range (IOR) for age, weight, body mass index, and body mass index-for-age $z$-score were 4 years (IQR, 1.3 to 8 years), $20.2 \mathrm{~kg}($ IQR, 10.2 to 26.6$), 15.9 \mathrm{~kg} / \mathrm{m}^{2}$ (IQR, 13.9 to $\left.17.3 \mathrm{~kg} / \mathrm{m}^{2}\right)$, and $-0.8(I Q R,-1.9$ to 0.4$)$, respectively. The demographic and anthropometric parameters of the participants are summarized in Table 1.

Table 2 shows the data for all methods of weight estimation: measures of bias, precision, and accuracy for the study participants in subgroups of weight, age, and habitus category. In general, the PAWPER XL-MAC method showed the best performance statistics, followed by the PAWPER XL tape, Mercy method, and Broselow tape. Fig. 3 shows the overall accuracy of each weight estimation system (in terms of PW10 and PW20) together with the desired targets for the accuracy outcome measures, as sug- 

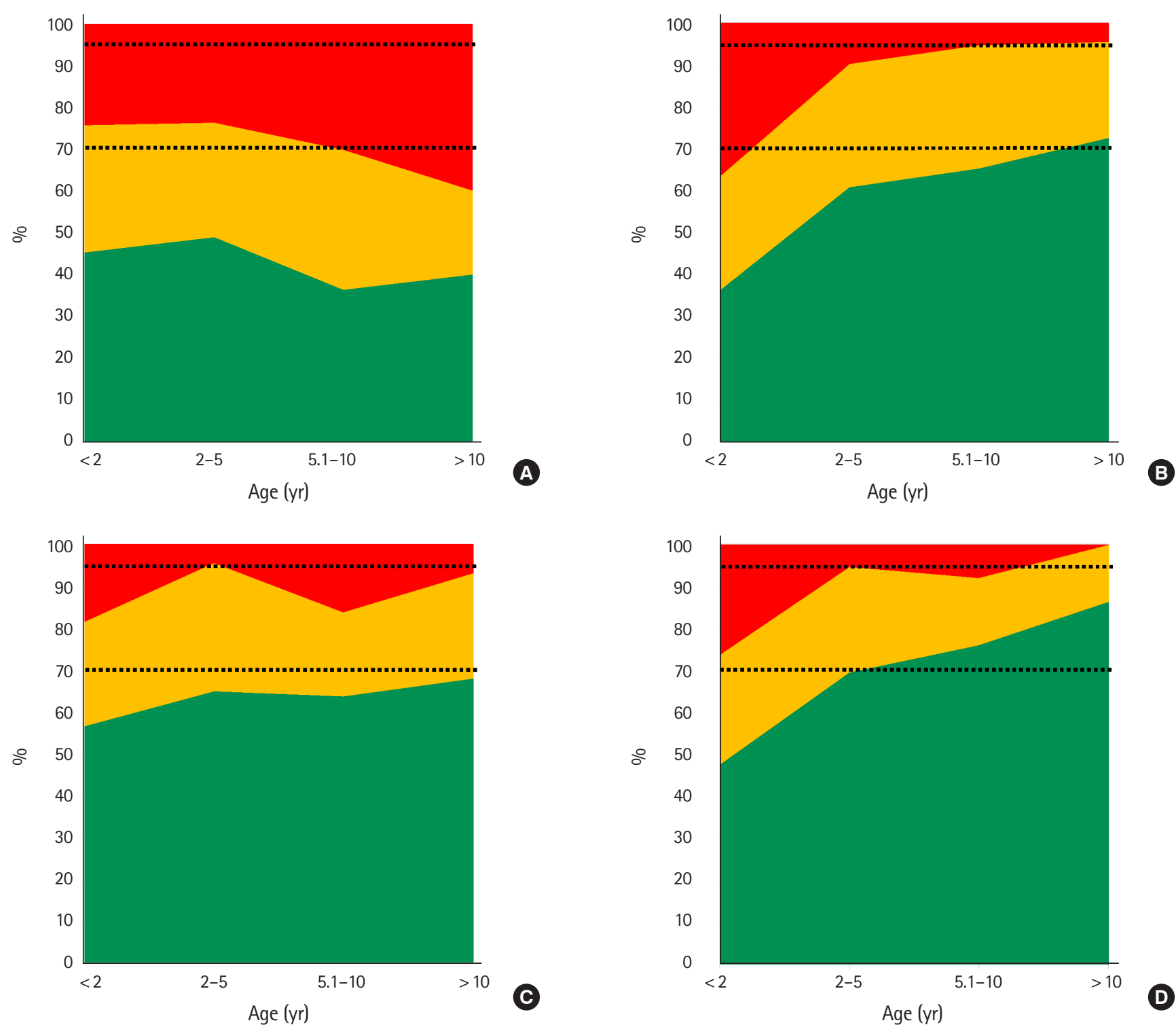

Fig. 3. Accuracy outcome data for the weight estimation systems by age group. The green section indicates the percentage of weight estimations within $10 \%$ of the actual weight. The red section indicates the percentage of weight estimations that exceed a $20 \%$ difference from the actual weight. The two dashed lines indicate previously proposed targets for accuracy: the green section (the percentage of weight estimations within 10\% of actual weight (PW10) should exceed 70\% (lower dashed line) and the red section should be above 95\% (upper dashed line). (A) Broselow tape, (B) Mercy method, (C) PAWPER XL tape, and (D) PAWPER XL mid-arm circumference method. PAWPER XL, pediatric advanced weight prediction in the emergency room extralong; PAWPER $X L$, pediatric advanced weight prediction in the emergency room extra-long.

gested previously. ${ }^{2}$ None of the systems achieved the desired benchmark accuracy standards in children below 2 years of age. The PAWPER XL-MAC method achieved the accuracy standards in children over 2 years of age and the Mercy method in children over 10 years of age.

Fig. 4 shows the analysis of time taken to perform the weight estimation for each method at the beginning and end of the study. Although all methods were used more quickly at the end of the study, only the PAWPER XL-MAC showed a substantial, but prob- ably clinically unimportant, reduction in time-to-weight estimation of just over $40 \%$.

Some participants' weights could not be estimated using the Broselow or Mercy methods. Forty-five children (15.0\%) were too tall for the Broselow tape (Table 3 shows the details of these children). One child (0.3\%) was too small for both the Mercy method and the Broselow tape. All participants could have their weight estimated by the PAWPER XL tape and the PAWPER XL-MAC method. 


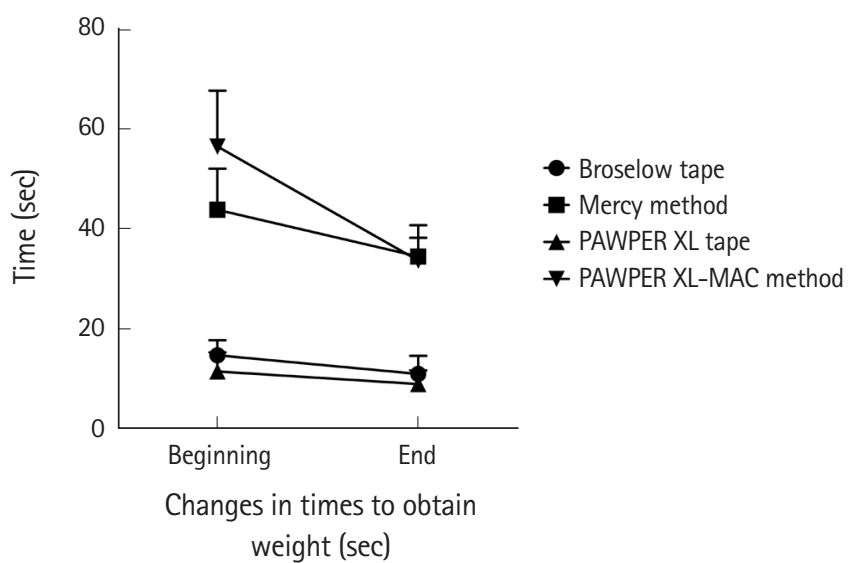

\begin{tabular}{lcclc}
\hline & $\begin{array}{c}\text { Broselow } \\
\text { tape }\end{array}$ & $\begin{array}{c}\text { PAWPER } \\
\text { XL }\end{array}$ & $\begin{array}{c}\text { PAWPER } \\
\text { XL-MAC }\end{array}$ & $\begin{array}{c}\text { Mercy } \\
\text { method }\end{array}$ \\
\hline $\begin{array}{l}\text { Difference in performance (sec) } \\
\text { \% improvement in perfor- }\end{array}$ & 5.1 & 2.1 & 24.8 & 6.5 \\
$\begin{array}{l}\text { mance } \\
\text { P for paired t-test }\end{array}$ & 34.2 & 19.2 & 43.2 & 15.9 \\
\hline
\end{tabular}

Fig. 4. An analysis of time taken to estimate weight in the first 10 and last 10 participants. The outcomes of the statistical analyses are shown in the data table. PAWPER $\mathrm{XL}$, pediatric advanced weight prediction in the emergency room extra-long; MAC, mid-arm circumference.

\section{DISCUSSION}

\section{Demographic characteristics of the study population}

This study population included a large number of young children (nearly $40 \%$ were under 2 years old) and a significant number of older children (a quarter of the children were older than 8 years). The sample included children at the age at which they are most likely to present with severe acute medical illnesses (typically under 5 years old) as well as older children who are more at risk for significant trauma presentations (typically over 10 years old). ${ }^{22}$ It was thus reasonably representative of the type of children who might require emergency weight estimations, despite the fact that no stratified sampling strategy was used.

The body habitus categories in this study were predominantly Habitus Score 2 (HS2) (18.7\%), HS3 (34.0\%), and HS4 (18.7\%). This was somewhat different from the initial PAWPER tape study, conducted in a population of higher economic status, where the children were assigned a body habitus of HS3 (51.4\%) and HS4 $(22.3 \%) .{ }^{23}$ In another South African study in a poorer community, Georgoulas et al. reported that HS were predominantly HS2 (38.0\%) and HS3 (30.7\%), similar to that found in the present study. ${ }^{13}$ Despite the fact that the population has a high prevalence of underweight children, there was also a significant incidence of overweight and obese children (20.0\%). This allowed for
Table 3. Details of the participants who were too tall for the Broselow tape

\begin{tabular}{lccl}
\hline Age $(\mathrm{yr})$ & Participants & Weight $(\mathrm{kg})$ & Height $(\mathrm{cm})$ \\
\hline 8 & $1(2.2)$ & $33.1(-)$ & $148(-)$ \\
10 & $5(11.1)$ & $31.6(29.1-32.0)$ & $147(145-155)$ \\
11 & $8(17.8)$ & $45.6(41.5-59.9)$ & $156(148-158)$ \\
12 & $9(20.0)$ & $36.7(35.2-41.2)$ & $151(148-154)$ \\
13 & $6(13.3)$ & $45.7(38.4-52.0)$ & $161(154-166)$ \\
14 & $3(6.7)$ & $62.5(53.4-71.5)$ & $162(160-167)$ \\
15 & $1(2.2)$ & $46(-)$ & $160(-)$ \\
16 & $5(11.1)$ & $51.4(49.8-57.3)$ & $160(153-166)$ \\
17 & $7(15.6)$ & $52.8(49.7-57.5)$ & $172(168-173)$ \\
All & $45(100)$ & $51.4(36.1-55.7)$ & $155(150-166)$ \\
\hline
\end{tabular}

Values are presented as number (\%) or median (interquartile range).

the weight estimation systems to be tested across a wide variety of ages and body habitus types.

\section{Comparison of the performance of the weight-estimation methods}

The one dimensional length-based Broselow tape (arguably the gold standard weight estimation method) had the greatest bias, lowest precision, and lowest overall accuracy, which showed its inferiority to the two dimensional (length- and habitus-based) methods. This can be attributed to the absence of body habitus considerations in the tape's design, as has been shown in previous systematic reviews. ${ }^{2,8}$ This edition of the Broselow tape, the 2011 edition A which is now 8 years old, was modified to limit the degree of underestimation in populations from high-income countries. Unfortunately, the accuracy has shown limited improvement in these children, but worsening accuracy in populations from low- and middle-income countries. . $^{14,24}$

In our study, the Broselow tape tended to overestimate weight in both underweight and "normal weight" categories of children, but it was the most accurate in overweight children (HS $>4$ ). This was expected, as the Broselow tape has been shown to overestimate weight in a lower socioeconomic setting. ${ }^{6}$ In a meta-analysis of the Broselow tape published in 2017, the pooled accuracy of Broselow tape was 54.7\% (PW10). The lowest accuracy was in the group with low income but has a high prevalence of underweight participants, in which the PW10 was 42.2\%. This was consistent with the result of this study in which the PW10 for underweight children was $43.9 \%{ }^{7}$ The use of weight adjustment of the Broselow tape using visual estimates of body habitus or measurements such as MAC or waist circumference may be needed for the tape to remain relevant. ${ }^{25,26}$

The PAWPER XL-MAC method was the most accurate of all the weight estimation methods. The PAWPER XL-MAC method out- 
performed the PAWPER XL in older and heavier children, thus suggesting that the PAWPER XL-MAC method is a more objective method than the PAWPER XL tape. Clinical gestalt may be less accurate in assessing habitus, especially in taller and heavier children: this is related to the so-called contraction bias, which is the tendency for an observer to underestimate the size of objects (or people) that are larger than average. ${ }^{27}$ The performance of the PAWPER XL and PAWPER XL-MAC methods both improved with increasing age and body habitus. The PAWPER XL-MAC method was the least accurate in youngest children where it overestimated weight. This may be at least partly attributed to the difficulty in obtaining MAC in younger children due to poor cooperation. The width of the PAWPER XL-MAC tape is $4 \mathrm{~cm}$, which limited its use to measure MAC in neonates and infants, as the tape was too broad to fit at the midpoint of the arm. In this case, a regular measuring tape had to be used. Compared to a regular measuring tape, the PAWPER XL tape is made of a sturdy material and does not conform to the natural curvature of the arm.

The performance of the PAWPER XL tape and the PAWPER XLMAC method in previously published studies is shown in Table 4. ${ }^{15,19,20,28-30}$ These studies, from both high- and low-socioeconomic status populations, showed a PW10 accuracy range of 71.5\% to $83.4 \%$ for the PAWPER XL tape and $79.0 \%$ to $81.9 \%$ for the PAWPER XL-MAC method, while in the present study, the PAWPER XL and PAWPER XL-MAC had an overall PW10 of $62.7 \%$ and $67.3 \%$, respectively. In the present study, both the PAWPER XL tape and the PAWPER XL-MAC method showed higher accuracy than the Broselow tape and the Mercy method, but they did not quite achieve the accuracy rate reported in literature of nearly $80 \%$ of estimations within 10\% of actual weight. This difference in accuracy may have been due to a difference in this study population and the proportion of neonates and infants analyzed. The fact that the accuracy of both these tapes was substantially higher in older children in this study (and more similar to the outcomes in other studies) suggests that the accuracy in the younger age group was a major contributor to the reduced overall accuracy. This needs to be further explored in future studies.

The discrepancy might also be caused by differences in the accuracy of habitus assessment or measurement of MAC between different users. This could possibly improve with standardized training and practice. Human factor or user errors have been shown to be important in weight estimation studies, and sufficient training and experience are essential when using any of the weight estimation systems. ${ }^{29,31}$ The better performance of the PAWPER XLMAC method than the PAWPER XL tape also suggested that the objectivity of the measurement of MAC for habitus assessment was superior to the subjective gestalt assessment used with the PAWPER XL tape. This was especially noticeable in underweight and obese children. These findings further support the value of the objective method of assessing habitus, with the provision that training in the use of the system is adequate. ${ }^{29,31}$

The Mercy method showed the least bias in older and thinner children, which was consistent with a previous Australian study, but the most bias in the youngest children. ${ }^{17}$ This was expected, as the accuracy of the Mercy method decreases in younger children. ${ }^{14}$ Furthermore, the Mercy method was used in the supine position in some of the infants, which may also have affected its accuracy as has been suggested previously. ${ }^{29}$ The Mercy device was originally designed for children 2 months to 16 years old, but in the present study, the Mercy method was used to estimate the weight of children from birth. The high degree of bias may, therefore, be due to the proportion of neonates included in the study $(n=63)$. However, in the present study, the Mercy method was still more accurate than the Broselow tape.

Table 4. Details of previously published studies on the PAWPER XL tape and PAWPER XL-MAC method

\begin{tabular}{|c|c|c|c|c|}
\hline Authors & Location & Method & Accuracy (\%) & Comments \\
\hline Wells et al. (2017) ${ }^{15}$ & South Africa & PAWPER XL & 83.4 & $\begin{array}{l}\text { This was the original validation study in a population with medium to high } \\
\text { socioeconomic status. }\end{array}$ \\
\hline Shrestha et al. $(2018)^{28}$ & Nepal & PAWPER XL & 71.5 & This was a study in a low socioeconomic population. \\
\hline Wells et al. $(2018)^{29}$ & South Africa & PAWPER XL & 73.0 & $\begin{array}{l}\text { This was a study during simulated paediatric emergencies to assess human } \\
\text { factor errors. }\end{array}$ \\
\hline Manyoni et al. (2019) $)^{30}$ & South Africa & PAWPER XL & 74.0 & This was a study in a very low socioeconomic population. \\
\hline Wells $(2019)^{20}$ & $\begin{array}{l}\text { Global low and middle-in- } \\
\text { come countries }\end{array}$ & PAWPER XL-MAC & 79.3 & $\begin{array}{l}\text { Study performed in datasets from a very large pool of anthropometric } \\
\text { studies from across the world. }\end{array}$ \\
\hline
\end{tabular}

This table does not include studies using the original PAWPER tape. The accuracy metric shown in this table refers to the percentage of weight estimations achieved within $10 \%$ of the actual weight. It is the most useful overall indicator of performance of a weight estimation system, and a value $>70 \%$ is considered to indicate very good accuracy.

PAWPER XL, pediatric advanced weight prediction in the emergency room extra-long; MAC, mid-arm circumference. 
Time taken to perform weight estimations in each method Weight estimations using the Broselow tape and PAWPER XL tape were significantly faster than using the methods that included a measurement of MAC. Both the Broselow and the PAWPER XL tape could provide an estimated weight within 15 seconds from the time of encounter. Methods incorporating the use of MAC took $>30$ seconds than the non-MAC methods in this study. This is potentially important in an emergency setting where time is limited, although there is no explicit evidence to confirm this, as times taken for weight estimation have not been previously studied in any form. The PAWPER XL-MAC system showed the most improvement with practice, with an overall improvement of 22.7 seconds (a $40 \%$ reduction of the initial time). Although this was a substantial decrease in time, the significance in a clinical setting would be uncertain. This finding suggests that training and practice can improve the efficiency in the use of the PAWPER XLMAC method and should be encouraged. In practice, the PAWPER $\mathrm{XL}$ tape could be used for rapid assessment of the body habitus in an urgent situation, and the PAWPER XL-MAC method could then be used to fine tune the measurement once the child was in a more stable condition. ${ }^{32}$ Only a single previous study has reported the times taken to obtain a weight estimation. ${ }^{29}$ The findings were very similar in terms of the times taken for weight estimation by a group of experienced emergency physicians.

\section{Restrictions to weight estimation capability}

The Broselow tape can provide weight estimation in children between 46 and $143 \mathrm{~cm}$ in length with a maximum weight estimation of $36 \mathrm{~kg}$. In the Broselow tape method, if a child exceeds the length of the tape, adult drug dosages and equipment should be used. ${ }^{3}$ Two studies, which examined children of appropriate age who exceeded the length of the Broselow tape, showed that the actual mean weight of this subgroup did not approximate that of adult weights. ${ }^{33,34}$ Many children in studies using the Broselow tape, often as many as one third of the study sample, had to be excluded, as they exceeded the length of the tape. ${ }^{7}$ Given the uncertainty of what should be done with children who exceed the length of the Broselow tape, this is a major flaw in the methodology..$^{33}$ In our study, 45 children (15\%) exceeded the length of the Broselow tape, and only five children above 11 years old were able to fit within the length constraints of the tape. Although the Broselow tape is recommended for use in children up to 12 years old, even in a resource-scarce setting, the tape cannot estimate a significant proportion of the children attending the facility. All children's weights could be estimated by the PAWPER XL and PAWPER XL-MAC methods, which was an advantage compared with the other methods in this study. It also overcomes the limitation of the original PAWPER tape in this respect.

This study has some limitations. Data collection was performed by a single researcher; therefore, no comparison of interobserver variability was possible. The clinical gestalt was based on the researcher's perception, which may vary from that of other healthcare workers depending on experience and training. This may have limited the accuracy of weight estimation in this study below what might be theoretically possible. Further studies with more than one data collector and blinding of the investigators to the results of the weight estimation methods as well as the actual weight may assist in eliminating variability and establishing inter-observer reliability.

This study population might be different from other populations, and the results may, therefore, not be generalizable to other populations. The difference in the performance of weight estimation systems in children of different habitus and age (in particular) means that the performance metrics will differ in populations with a different demographic composition. Stratified or balanced sampling was not used to endure equal numbers of children in each age and weight category, which could potentially have introduced some bias.

In summary, the PAWPER XL-MAC method and the PAWPER XL tape were the most accurate weight estimation systems, whereas the Broselow tape had the worst performance. The PAWPER XL tape required the shortest time to generate a weight estimate, while the PAWPER XL-MAC method showed the most improvement with practice over the course of the study. The Broselow tape failed to provide weight estimation in a substantial number of children, which was a further limitation of its use. Overall, the PAWPER $X L$ tape and the PAWPER XL-MAC method were the best performers when both accuracy and reasonable speed were considered.

Future research on the inter-observer reliability of the PAWPER XL-MAC method would be useful, as well as an assessment of the performance of this method in distinct subsets of children in different weight-status and age categories.

\section{CONFLICT OF INTEREST}

Professor Wells is the developer of the PAWPER tape, the PAWPER XL tape and the PAWPER XL-MAC system but derives no financial benefit from them. Except for that, no potential conflict of interest relevant to this article was reported.

\section{REFERENCES}

1. Kleinman $M E$, Chameides L, Schexnayder SM, et al. Pediatric advanced life support: 2010 American Heart Association guide- 
lines for cardiopulmonary resuscitation and emergency cardiovascular care. Pediatrics 2010;126:e1361-99.

2. Wells M, Goldstein LN, Bentley A. The accuracy of emergency weight estimation systems in children-a systematic review and meta-analysis. Int J Emerg Med 2017;10:29.

3. Lubitz DS, Seidel JS, Chameides L, Luten RC, Zaritsky AL, Campbell FW. A rapid method for estimating weight and resuscitation drug dosages from length in the pediatric age group. Ann Emerg Med 1988;17:576-81.

4. Nieman CT, Manacci CF, Super DM, Mancuso C, Fallon WF Jr. Use of the Broselow tape may result in the underresuscitation of children. Acad Emerg Med 2006;13:1011-9.

5. Bourdeau S, Copeland J, Milne WK. Accuracy of the Broselow tape in estimating the weight of First Nations children. Can J Rural Med 2011;16:121-5.

6. Clark MC, Lewis RJ, Fleischman RJ, Ogunniyi AA, Patel DS, Donaldson RI. Accuracy of the Broselow tape in South Sudan, "The hungriest place on earth". Acad Emerg Med 2016;23:21-8.

7. Wells M, Goldstein LN, Bentley A, Basnett S, Monteith I. The accuracy of the Broselow tape as a weight estimation tool and a drug-dosing guide: a systematic review and meta-analysis. Resuscitation 2017;121:9-33.

8. Wells $M$, Goldstein LN, Bentley A. A systematic review and meta-analysis of the accuracy of weight estimation systems used in paediatric emergency care in developing countries. Afr J Emerg Med 2017;7(Suppl):S36-54.

9. Wells $M$, Barnes L, Vincent-Lambert C. Paediatric weight estimation practices of advanced life support providers in Johannesburg, South Africa. Afr J Emerg Med 2018;8:51-4.

10. Young KD, Korotzer NC. Weight estimation methods in children: a systematic review. Ann Emerg Med 2016;68:441-51.

11. Abdel-Rahman SM, Paul IM, James LP, Lewandowski A; Best Pharmaceuticals for Children Act-Pediatric Trials Network. Evaluation of the Mercy TAPE: performance against the standard for pediatric weight estimation. Ann Emerg Med 2013; 62:332-9.

12. Dicko A, Alhousseini ML, Sidibe $B$, Traore M, Abdel-Rahman SM. Evaluation of the Mercy weight estimation method in Ouelessebougou, Mali. BMC Public Health 2014;14:270.

13. Batmanabane G, Jena PK, Dikshit R, Abdel-Rahman S. Using the Mercy method for weight estimation in Indian children. Glob Pediatr Health 2015;2:2333794X14566625.

14. Georgoulas VG, Wells M. The PAWPER tape and the Mercy method outperform other methods of weight estimation in children at a public hospital in South Africa. S Afr Med J 2016; 106:933-9.

15. Wells M, Goldstein L, Bentley A. A validation study of the PAW-
PER XL tape: accurate estimation of both total and ideal body weight in children up to 16 years of age. Trauma Emerg Care 2017;2:1-8.

16. Wells $M$, Goldstein LN, Bentley A. The use of body habitus reference images improves the ability of novices to accurately estimate children's weight using the PAWPER XL tape system. J Emerg Med 2018;54:165-75.

17. O'Leary F, John-Denny B, McGarvey K, Hann A, Pegiazoglou I, Peat J. Estimating the weight of ethnically diverse children attending an Australian emergency department: a prospective, blinded, comparison of age-based and length-based tools including Mercy, PAWPER and Broselow. Arch Dis Child 2017; 102:46-52.

18. Cattermole GN, Leung M, Mak P, So HK, Graham CA, Rainer TH. Children's weights correlate more strongly with mid-arm circumference (MAC) than with age, height or foot-length. J Emerg Med 2009;37:228-9.

19. Wells $M$, Goldstein LN, Bentley A. Development and validation of a method to estimate body weight in critically ill children using length and mid-arm circumference measurements: the PAWPER XL-MAC system. S Afr Med J 2017;107:1015-21.

20. Wells M. A validation of the PAWPER XL-MAC tape for total body weight estimation in preschool children from low- and middle-income countries. PLoS One 2019;14:e0210332.

21. Abdel-Rahman SM, Ridge AL. An improved pediatric weight estimation strategy. Open Med Dev J 2012;4:87-97.

22. Downing A, Rudge G. A study of childhood attendance at emergency departments in the West Midlands region. Emerg Med J 2006;23:391-3.

23. Wells M, Coovadia A, Kramer E, Goldstein L. The PAWPER tape: a new concept tape-based device that increases the accuracy of weight estimation in children through the inclusion of a modifier based on body habitus. Resuscitation 2013;84:22732.

24. Yamamoto $L G$, Inaba $A S$, Young $L L$, Anderson KM. Improving length-based weight estimates by adding a body habitus (obesity) icon. Am J Emerg Med 2009;27:810-5.

25. Tanner D, Negaard A, Huang R, Evans N, Hennes H. A prospective evaluation of the accuracy of weight estimation using the Broselow tape in overweight and obese pediatric patients in the emergency department. Pediatr Emerg Care 2017;33: 675-8.

26. Wells M, Goldstein L, Bentley A. Accuracy of weight estimation by the Broselow tape is substantially improved by including a visual assessment of body habitus. Pediatr Res 2018;83: 83-92.

27. Robinson E. Overweight but unseen: a review of the underes- 
timation of weight status and a visual normalization theory. Obes Rev 2017;18:1200-9.

28. Shrestha K, Subedi P, Pandey O, Shakya L, Chhetri K, House DR. Estimating the weight of children in Nepal by Broselow, PAWPER XL and Mercy method. World J Emerg Med 2018;9: 276-81.

29. Wells M, Goldstein LN, Bentley A. The accuracy of paediatric weight estimation during simulated emergencies: the effects of patient position, patient cooperation, and human errors. Afr J Emerg Med 2018;8:43-50.

30. Manyoni MJ, Goldstein LN, Wells M. A comparison of four weight estimation systems for paediatric resuscitation. S Afr J Surg 2019;57:40-6.

31. Abdel-Rahman SM, Jacobsen R, Watts JL, et al. Comparative performance of pediatric weight estimation techniques: a human factor errors analysis. Pediatr Emerg Care 2017;33:54852.

32. Wells M, Goldstein LN. An algorithm to improve the accuracy of emergency weight estimation in obese children. Pan Afr Med J 2018;31:90.

33. Cattermole GN, Leung PY, Graham CA, Rainer TH. Too tall for the tape: the weight of schoolchildren who do not fit the Broselow tape. Emerg Med J 2014;31:541-4.

34. Lowe CG, Campwala RT, Ziv N, Wang VJ. The Broselow and Handtevy resuscitation tapes: a comparison of the performance of pediatric weight prediction. Prehosp Disaster Med 2016;31:364-75. 